\title{
Deux Monolistrini nouveaux (Crustacea, Isopoda) des eaux souterraines de Croatie
}

\author{
par Christa L. Deeleman-Reinhold 1)
}

avec planche $65(1)-67$ (3)

Au cours de ces dernières années le nombre de Monolistrini connus des Alpes Dinariques de Yougoslavie s'est rapidement accru grâce aux recherches persévérantes, entreprises par B. Sket (Ljubljana) et ses collaborateurs. Depuis la parution de la Monographie de Karaman (1954), le nombre d'espèces et sous-espèces connues de Yougoslavie est passé de neuf à dixhuit, ce qui fait qu' actuellement les Monolistrini se répartissent en vingt-six espèces dont cinq habitent les Alpes méridionales de l'Est de l'Italie et trois la France.

Le manuscrit de la Monographie des Monolistrini des Alpes orientales et de la chaîne dinarique par B. Sket (1964-1965) était à peine achevé, qu'une nouvelle forme fut découverte dans une grotte de Croatie. Cette forme, qui sera décrite ci-dessous, comme sous-espèce de Monolistra caeca, appartient au sous-genre Monolistra Gerstaecker, 1856 s. str., ayant une pince préhensile au péréiopode II du mâle et des uropodes bien développés. Elle se sépare de $M$. caeca Gerstaecker, par plusieurs caractères importants. De plus, en 1965 une espèce nouvelle de Microlistra fut rencontrée dans une autre grotte croate, à environ $30 \mathrm{~km}$ de la première. Cette espèce intéressante présente une série de caractères intermédiaires entre les espèces épineuses, $M$. spinosa Racovitza, M. spinosissima Racovitza et $M$. bolei Sket d'une part et les espèces non épineuses $M$. schottlaenderi (Stammer) et M. pretneri Sket d'autre part.

Nous devons l'obligeance de R. Kevo du Zavod za zaštitu prirode (l'Institut pour la protection de la nature) de Zagreb d'avoir pu effectuer des récoltes dans les grottes de Croatie. Nous exprimons aussi notre gratitude à J. Jansen (Rijswijk) et A. A. Mabelis (Leiden) qui nous ont accompagné en 1965 et à B. Sket (Ljubljana) qui a bien voulu nous envoyer quelques exemplaires de Microlistra spinosa.

1) Minckelersweg 18, Hoogerheide, Pays-Bas Aprés 1-3-1969. Sparrenlaan 8, ossendrecht, Pays-Bas. 


\section{Monolistra caeca caeca Gerstaecker 1856}

Matériel. -

Sopot pećina, Severin na Kupi, Croatie. Grotte située directement au bord de la Kolpa, à la limite septentrionale de la Croatie. De nombreux exemplaires, récoltés le 3 août 1966 par Mlle M. Dekking et A. P. B. Deeleman.

- Source «Vrelo Okrugljak», Velika Jadić, à quelques kilomètres de la station précédente. Les Isopodes étaient exposés directement à la lumière du jour. De nombreux exemplaires récoltés le 3 juillet 1966 par P. R. et C. L. Deeleman et Mlle M. Dekking.

- Grotte de la "Vrelo Rudnica», Kamenica, province de Slunj. Dans un lac, habité aussi par Niphargus sp. et Typhlogammarus mrazeki Karaman, 10 juillet 1966 (P. R. et C. L. Deeleman).

Ces exemplaires correspondent très bien à la description de Monolistra caeca, donnée par Racovitza. (1910)

\section{Monolistra (Monolistra) caeca meridionalis n. subsp.}

Matériel. - Grotte Crna pećina, Novi Kršlija, Rakovica, Yougoslavie; à environ 20 mètres de l'entrée dans un lac dont l'eau dessert une source située quelques mètres en aval de l'entrée de la grotte : cette source alimente un ruisseau qui débouche dans la Korana. Les Monolistra vivaient en compagnie de nombreux Orniphargus croaticus; altitude $450 \mathrm{~m}$; 7 et 8 août 1964: $4{ }^{\star}$, 1 우 non ovigère et 6 non adultes, leg. P. R. Deeleman; $1^{\mathrm{er}}$ et 11 juillet $1965: 11 \delta^{\star}$, et 1 o et 1 ex. non adulte, leg. P. R. Deeleman.

- Grotte «Pećina Jezero», située entre Seča et Ručiće; Barilović, non loin de la Korana, province de Karlovac. De nombreux exemplaires dans un lac à eau stagnante, sur le fond couvert d'argile, récoltés le 2 août 1966 (P. R. et C. L. Deeleman).

- Grotte près de Tržić, $5 \mathrm{~km}$ à l'ouest de Primišlje, province de Slunj. La grotte est située directement au bord de la rivière Mrežnica. De nombreux exemplaires dans un lac à eau stagnante (8 juillet 1966: P. R. Deeleman et Mlle M. Dekking). L'holotype, un o de $11 \mathrm{~mm}$ et quelques paratypes se trouvent dans la collection du Rijksmuseum van Natuurlijke Historie, Leiden, no. Crust. I 1642, 1643, les autres paratypes se trouvent dans notre collection.

Description. - Les mâles mesurent 10 à $12 \mathrm{~mm}$, les femelles $8 \mathrm{~mm}$. Les exemplaires de la grotte de la Mrežnica sont un peu plus petits. La largeur au IV ${ }^{\text {ième }}$ péréionite est la moitié de la longueur du corps.

La carapace, couverte de nombreuses petites gibbosités est glabre ou partiellement couverte de quelques poils peu abondants. Les antennes I 
mesurent de $1 / 4$ à $2 / 9$ de la longueur du corps; le fouet est composé de 7 ou 8 articles et porte respectivement une lame olfactive sur les articles III, IV, et VI ou IV, V et VII chez un exemplaire il y a des lames olfactives aux articles III, IV, V et VII. Les antennes II sont $4 / 3$ fois plus longues que les antennes I, le fouet est formé de 10-12 articles. Les apophyses dentaires gauche et droite des mandibules portent 4 dents bien développées, l'apophyse interne gauche porte 3 dents.

Le premier péréionite est de $1 / 6$ moins large que le quatrième. La surface articulaire de l'épimère est large, triangulaire, plane et bordée d'une crête longeant le bord intérieur, la forme de ce triangle est variable au sein de l'espèce; celle de l'épimère II est trapézoidale; les épimères des autres péréionites sont lamellaires, plus ou moins arrondis.

Chez les mâles le basipodite du péréiopode I est aplati et élargi, 3 fois plus long que large; celui du péréiopode II est $4 \frac{1}{2}$ plus long que large; chez la femelle ces rapports sont respectivement 3 et $5 \frac{1}{2}$. Le bord sternal du propodite I est garni de 4 ou 5 tiges pennées chez le $\sigma^{\dagger}$, de 5 chez la $q$, les deux distales étant juxtaposées à l'extrémité.

Le péréiopode II du $\delta^{\star}$ est transformé en pince, son bord inférieur porte une protubérance effilée, aiguë, qui s'écarte du grand axe de l'article suivant une direction oblique. La lame au bord inférieur du dactyle est de forme triangulaire et bien plus large que le dactyle lui-même.

Tous les péréiopodes sont longs et minces; les ongles sont très longs $(1 / 2$ à $2 / 3$ de la longueur du dactyle) et faiblement courbés. Il n'y a pas de différence sexuelle dans la forme des dactyles. Sur les basipodites des péréiopodes III, IV, V et chez certains exemplaires sur ceux des péréiopodes VI, il y a deux soies sensorielles sur la face antérieure: une dans la région proximale l'autre près de l'extrémité. Les péréiopodes VII mesurent la moitié de la longueur du corps; les basi- et ischiopodite sont 8 fois, le méropodite 3 fois, le carpopodite 4 fois, le propodite 5 fois et le dactyle $2 \frac{1}{2}$ fois plus longs que larges. Chez les exemplaires de Barilović, les pattes sont moins grêles.

Sur le propodite du pléopode I se trouvent 2 ou 3 épines sur le bord interne. L'exopodite est ellipsoïde, $5 / 3$ fois plus long que large, avec des tiges ciliées aux bords latéraux et distal dont le nombre varie entre 11 et $19 \mathrm{chez}$ les 5 individus examinés, chez la + il y en a 14 ; l'endopodite est de $1 / 7$ plus long que l'exopodite, à bords parallèles; $3-4$ tiges s'insèrent sur le bord distal.

L'exopodite et l'endopodite du pléopode II sont subquadrangulaires; l'endopodite est $5 / 4$ fois plus long que l'exopodite et à peine plus long que l'organe copulateur; l'exopodite est 2 fois plus long que large, avec 14-24 tiges ciliées sur le bord de la partie distale; l'article est souvent plié pour s'adapter à la forme de la cavité du telson; l'endopodite est environ $21 / 4$ plus long que large, son bord distal est muni de 9-15 tiges ciliées. 
L'exopodite III est ovalaire, sa longueur n'atteint pas le double de sa largeur. Chez M. c. caeca, la longueur est au moins le double de la largeur. Sur son bord extérieur, près de la base, se trouvent quelques soies. L'endopodite est subquadrangulaire, environ $1 / 10$ moins long que l'exopodite et de la même largeur. L'exopodite IV est subovoïde, à peine moins long que l'exopodite III; l'aire respiratoire s'étend sur un peu plus de la moitié du bord interne, l'endopodite IV est aussi long et aussi large que l'exopodite. L'exopodite $\mathrm{V}$ est irrégulièrement elliptique, à sommet anguleux, un peu plus long que l'exopodite IV et un peu moins large; l'aire respiratoire est ovoïde et s'étend sur les $2 / 7$ de la longueur de l'article; l'endopodite est subovalaire à sommet anguleux, $4 / 5$ de la longueur et $8 / 9$ de la largeur de l'exopodite.

Le pléotelson atteint environ un tiers de la longueur totale du corps; le pléonite est aussi large que la partie la plus large du péréion, au IV ième segment. La partie antérieure des épimères du pléonite forme une dépression nette, dans laquelle se logent les épimères VII pendant l'enroulement. Cette dépression est éloignée du bord latéral de l'épimère; ce bord est arrondi, non anguleux.

La région médiane caudale du telson présente une bosselure subconique dont l'extrémité postérieure se trouve au niveau du bord sterno-caudal du telson ou le déborde faiblement. Le bord sterno-caudal est évasé; la crête qui se trouve juste au-dessus du bord à l'extérieur et contre laquelle s'appliquent les uropodes lors de l'enroulement, est bien développée, saillante et aiguë.

Les uropodes sont aplatis, anguleux, légèrement recourbés vers l'intérieur, 6-7 fois plus longs que larges; quand ils sont appliqués contre le bord postérieur du telson, ils se croisent sur un tiers de leur longueur.

Monolistra caeca meridionalis se distingue nettement de la forme typique: par la forme de la pince préhensile du ơ dont la protubérance au propodite est très effilée tandis que la lame du dactyle atteint une dimension plus considérable; par le plus grand nombre de tiges sur l'endopodite du pléopode I; par l'exopodite du pléopode III dont la largeur excède la moitié de sa longueur et par ses uropodes plus courts et gros, moins courbés.

\section{Microlistra sketi $\mathrm{n} . \mathrm{sp}$.}

Matériel. - Grotte près de Pećina Selo, (Lešće) près de la route OtočacGospić, Croatie, Yougoslavie. Pendant la saison humide, de l'eau surgit de la grotte et afflue à la Gacka. La grotte est située au pied de la bordure septentrionale du plateau de la Lika.

Dans cette grotte très humide, dont le sol est recouvert entièrement d'une couche épaisse de limon très glissant se trouvent plusieurs lacs profonds en 
forme d'entonnoir, habités par Orniphargus kolombatovici Karaman et quelques lacs moins profonds et plus plats. Les Microlistra étaient tous rassemblés sur les parties immergées de la paroi où la roche vient à nu et ils semblaient éviter le limon. Dans ces mêmes lacs nageaient quelques Atyides Troglocaridella hercegovinensis Babić 1922 (det. L. B. Holthuis). Altitude $450 \mathrm{~m} ; 28$ juillet $1965: 3{ }^{\star}$ et 18 o , dont une portait 11 oeufs; leg. P. R. Deeleman, A. A. Mabelis et J. Jansen; 31 juillet 66 : nombreux exemplaires (C. L. Deeleman, P. R. et A. P. B. Deeleman). L'holotype, un ơ de $9 \mathrm{~mm}$, l'allotype, une $q$ de $10 \mathrm{~mm}$ et quelques paratypes se trouvent dans la collection du Rijksmuseum van Natuurlijke Historie à Leiden, no. Crust. I (1644, 1645 et 1646), les autres exemplaires sont dans notre collection.

Description. - La longueur du corps des exemplaires mâles est respectivement de: 8,5, 9,0 et 9,5 mm; celle des femelles entre 8 et $12 \mathrm{~mm}$, leur taille moyenne étant plus grande que celle des mâles. La plus grande largeur du corps, au IVième péréionite et au pléonite, représente $3 / 7$ de sa longueur.

La tête est munie de deux bosses sur sa marge caudale. Tous les péréionites sont pourvus de 5 séries longitudinales de bosses: une médiane, deux médio-latérales et deux latérales; le pléonite est dépourvu de bosses, le telson porte un nombre indéfini de petites bosses rangées sans ordre apparent ou en deux lignes médio-latérales irrégulières. Sur l'épimère VI s'implante une épine conique, recourbée caudalement. Dans la région rostrale du telson se trouve de chaque côté une épine latérale, implantée devant l'uropodite, plus longue que celle du péréionite VI et également recourbée caudalement, d'une longueur assez variable, atteignant chez quelques exemplaires presque le bord caudal du telson. La bosselure médiane caudale du telson se termine postérieurement par deux épines coniques médio-latérales, un peu plus courtes que les épines antérieures du telson, recourbées légèrement vers le côté interne. Des différences sexuelles dans l'armature de la carapace n'ont pas été constatées.

Les antennes I atteignent le $2 / 7$ de la longueur du corps. Le fouet possède 7-10 articles, le plus souvent 8 , les lamelles olfactives manquent sur les deux premiers articles, l'antépénultième et le dernier. Les antennes II mesurent le $5 / 4$ de la longueur des antennes I, leur fouet est formé de 10 à 14 articles. Les apophyses dentaires des mandibules sont en forme de spatules non dentées, les maxillipèdes de la femelle ovigère portent un lobe externe.

La largeur du premier péréionite est presque égale à celle du quatrième, la surface articulaire de son épimère est allongée, ovale-triangulaire, le bord extérieur est presque droit, l'angle caudal-externe tronqué-arrondi; chez les épimères II et III la surface articulaire est présente, mais petite. Les épimères IV à VII se terminent en pointe aiguë très légèrement soulevée vers l'extérieur; tous les épimères sont dirigés presque verticalement vers le bas. La largeur du septième péréionite est environ égale à celle du premier. Les 
différences sexuelles dans la largeur du basipodite I que Racovitza a observé chez $M$. spinosa et $M$. spinosissima ne sont pas distinguables chez notre espèce.

Le péréiopode I est $31 / 2$ fois plus court que la longueur du corps; le propode porte $5-7$ tiges pennées sur sa face sternale, le carpe en a deux. La longueur du péréiopode II atteint le $6 / 5$ du péréiopode I, le péréiopode VII mesure un peu plus de la moitié du corps. Sur les basipodites des péréiopodes I à VII se trouvent une longue soie sensorielle au milieu du bord externe et 3-4 soies identiques sur la partie distale du bord antéro-externe; elles sont plus agrégées que celles de Microlistra spinosa; elles manquent sur les péréiopodes I et II. Une soie ciliée se trouve tergalement sur chacune des extrémités distales des protopodites II à VII.

Les dactyles des mâles sont plutôt cylindriques, tandis que ceux des femelles sont plus acuminés. Les pattes des femelles sont plus grêles que celles des mâles. La longueur du propode VII par exemple est chez la o 6 fois, chez le $\sigma^{\star} 5$ fois la largeur.

Les pléopodes sont caractéristiques du genre. Le pléopode I est composé d'un protopodite à 3 épines, un exopodite ovale, légèrement atténué du côté distal, avec 5-8 tiges pennées sur le bord distal et un endopodite à bords parallèles ou légèrement atténués, droit ou incliné vers l'extérieur, aussi long que l'exopodite, le bord distal avec 1-3 tiges pennées; les pléopodes II ont l'exopodite semi-ovale, un peu moins long que l'endopodite, à bord externe plus ou moins convexe, le bord interne plutôt subrectiligne, pourvu de 12-17 tiges ciliées sur le bord distal. L'endopodite II est de forme subquadrangulaire et s'élargit légèrement vers le sommet; les bords de la région distale sont pourvus de $18-25$ tiges ciliées. L'organe copulateur est un peu plus long que l'endopodite, l'apex est recourbé vers le côté sternal. L'exopodite III est subquadrangulaire, 2 fois plus long que large; quelques sètes se trouvent proximalement sur le bord extérieur. L'endopodite est subquadrangulaire, aussi large que l'exopodite et environ de $1 / 10$ moins long. L'exopodite IV est aussi long et aussi large que l'exopodite III, ovalaire à sommet atténué; l'aire respiratoire occupe plus de la moitié du bord interne; l'endopodite est aussi long et large que l'exopodite, ovaleelliptique et légèrement atténué dans sa partie distale. L'exopodite $\mathrm{V}$ est irrégulièrement elliptique à sommet anguleux, un peu plus long et d'un quart moins large que l'exopodite IV, l'aire respiratoire comprend environ un tiers de la longueur et la moitié de la largeur de l'article; l'endopodite est elliptique à sommet anguleux, aussi large et un peu moins long que l'endopodite.

Le pléotelson représente environ un tiers de la longueur du corps. Les angles rostraux-externes des épimères du pléonite I sont arrondis, les bords caudaux-externes aigus; les limites de la dépression rostrale sont mal définies, 
la région caudale des épimères est largement recourbée vers l'extérieur et constitue une marge horizontale plane.

La bosselure du telson déborde fortement le bord sterno-caudal du telson, comme chez les autres Microlistra; le bord entre les deux épines caudales est droit, la longueur des épines latérales et caudales est variable et n'a pas de rapport apparent avec le sexe de l'animal. Les uropodites sont construits de la même façon que ceux des autres Microlistra.

Microlistra sketi est la sixième espèce du genre; sa localisation géographique se situe entre celle de $M$. spinosa Racovitza, $M$. spinosissima Racovitza et $M$. bolei Sket (tous les trois habitent la Slovénie) et celle de M. pretneri Sket de Dalmatie moyenne; elle offre un intérêt spécial parce que, dans la conformation et le nombre d'épines de la carapace et l'acuité des épimères elle représente un intermédiaire jusqu'ici inconnu entre les formes épineuses de la Slovénie citées ci-dessus et les formes non-épineuses, tuberculeuses (M. schottlaenderi et M. pretneri).

De plus si tous les autres représentants du genre ont été trouvés jusqu'à maintenant, dans des eaux plus ou moins courantes des grottes et sources (Sket 1964, p. 182), notre espèce, elle, a été recueillie dans de l'eau tout à fait stagnante, où elle était abondante.

octobre 1967

\section{RÉSUMÉ}

Description de Monolistra (Monolistra) caeca meridionalis nov. subsp. Cette sous-espèce a été recueillie dans trois grottes de la Croatie du Nord, Yougoslavie. Elle se distingue de $M$. c. caeca e. a. par la forme de la protubérance du propode du péréiopode II du $\delta^{\lambda}$, qui est effilée et aiguë, par les endopodites des pléopodes I portant 3-4 tiges $(1-2$ chez M. c. caeca), par les endopodites des pléopodes III plus larges et par ses uropodes moins longs et moins courbés. Dans la région prospectée 3 stations de M. c. caeca et 3 stations de M.c. meridionalis ont été découvertes.

L'autre espèce nouvelle, Microlistra sketi est la sixième espèce du genre et a été recueillie dans une grotte, dans l'eau d'une source périodique de la Gacka, Croatie. Celle-ci présente quelques caractères intermédiaires entre les espèces épineuses habitant la Slovénie et les espèces non épineuses, tuberculeuses, M. pretneri Sket de Dalmatie et M. schottlaenderi (Stammer) des environs de Trieste, ayant trois paires d'épines relativement courtes sur la carapace.

\section{SUMMARY}

A new subspecies and a new species are described: Monolistra (Monolistra) caeca meridionalis nov. subspec. was found in three caves in the northern part of Croatia, Yougoslavia. It is distinguishable from the typical form principally by the thin, acuminate form of the protuberance of the protopodite of the IInd male peraeopod, by the endopodite of the Ist pleopod which bears 3-4 setae (1-2 in M. c. caeca), by the somewhat wider endopodite of the IIIrd pleopod and by the shorter, only slightly curved uropods. A sketch shows the situation of 6 newly discovered localities in northern Croatia, 3 of the typical form and three of the new subspecies.

Microlistra sketi is the $6^{\text {th }}$ species known of its genus and lives in a cave, in stagnant water of a periodic spring, tributary to the river Gacka in Croatia. The number (three pairs), and the length of the spines of the carapace and the pointed- 
ness of the epimers of the pereion are intermediate between those of the spiny forms living in Slovenia and the tubercular species M. pretneri Sket from Dalmatia and M. schottlaenderi (Stammer) from the vicinity of Trieste.

\section{BIBLIOGRAPHIE}

Karaman, St. (1954) - Über die Jugoslawischen Arten des Genus Monolistra (Isop.) Acta Mus. Macedonici Sci. Nat. II: 125-143.

Racovitza, E. G. (1910) - Sphéromiens et révision des Monolistrini. Arch. Zool. exp. et gén. 5 (IV): $625-758$.

- (1929) - Microlistra spinosa, n. g., n. sp., Isopode Sphéromien cavernicole nouveau de Slovénie. Bull. Soc. Sc. Cluj, IV.

- 1929 - Microlistra spinosissima n. sp. Isopode Sphéromien cavernicole nouveau de Slovénie. Ibid.

SKET, B. (1960) - Einige Formen der Malacostraca aus Jugoslawien III. Bull. Scient. 5 (3): $73-75$.

- (1961) - K problematiki naših Monolistrinov. II Jugoslavenski spel. Kongr. 1958: $171-178$.

- (1964) - Östliche Gruppe der Monolistrini, I. Systematischer Teil. Int. Journ. Spel. I $(1+2): 163-189$.

- (1965) - Östliche Gruppe der Monolistrini, II. Biologischer Teil. ibid., I (3): $249-267$.

STAMmer, H. J. (1930) - Eine neue Höhlensphäromide aus der Karst, Monolistra (Typhlosphaeroma) schottlaenderi. Zool. Anz. 88: 291-304.

\section{EXPLICATION DES PLANCHES 1 (1) - 3 (3)}

\section{PLANCHE 1 (1): MONOLISTRA CAECA MERIDIONALIS}

a) $O^{\star}$ de $12,3 \mathrm{~mm}$ : pléopode II gauche, $\times 20$

b) ${ }^{\wedge}$ de $12,3 \mathrm{~mm}$ : pléopode I gauche, $\times 20$

c) $o^{\star}$ de $11 \mathrm{~mm}$ (holotype): pléopode III $\times 10$

d) $\sigma^{\wedge}$ de $11 \mathrm{~mm}$ (holotype): pléopode IV $\times 10$

e) $0^{\star}$ de $11 \mathrm{~mm}$ (holotype): pléopode $\mathrm{V} \times 10$

f) $0^{t}$ de 12,3 mm: vue latérale gauche de l'animal enroulé $\times 10$

g) $\sigma^{x}$ de $11 \mathrm{~mm}$ : vue dorsale de la partie caudale du corps $\times 10$

h) $\sigma^{\star}$ de $12,3 \mathrm{~mm}$ : péréiopode II gauche, face caudale $\times 35$

i) $\sigma^{\pi}$ de $12,3 \mathrm{~mm}$ : péréiopode VII, $\times 10$

j) $\sigma^{\pi}$ de 12,3 mm: péréiopode I gauche, face caudale, $\times 35$

k) $\sigma^{\top}$ de $11 \mathrm{~mm}$ (holotype): vue de dessous de la partie gauche de la tête et des 3 premiers somites $\times 10$

\section{PLANCHE 2 (2): MICROLISTRA SKETI}

a) ㅇ de $10 \mathrm{~mm}$ (cotype) vue dorsale $\times 10$

b) $\uparrow$ de 12,5 mm: vue latérale de la partie caudale du corps, $\times 10$

c) + de $12,5 \mathrm{~mm}$ : pléopode I droite, $\times 20$

d) q de $12,5 \mathrm{~mm}$ : pléopode II droite, $\times 20$

e) $\delta^{t}$ de $9 \mathrm{~mm}$ (holotype) : pléopode III gauche, $\times 35$

f) $\sigma^{\top}$ de $9 \mathrm{~mm}$ (holotype): pléopode IV gauche $\times 35$

g) ot de $9 \mathrm{~mm}$ (holotype): pléopode $\mathrm{V}$ gauche $\times 35$

\section{PLANCHE 3 (3): MICROLISTRA SKETI}

a) $q$ de $12,5 \mathrm{~mm}$ : vue de dessous de la partie droite de la tête et des 3 premiers somites $\times 10$

b) + de $12,5 \mathrm{~mm}$ : vue latérale de la partie droite de la tête et premier péréionite, vues de gauche $\times 10$ 
c) $q$ de $12,5 \mathrm{~mm}$ : antenne I droite $\times 35$

d) + de $11 \mathrm{~mm}$ : partie gauche du corps, vue latéro-caudale $\times 10$

e) $\sigma^{7}$ de $9 \mathrm{~mm}$ (holotype): péréiopode I droite, race caudale, $\times 35$

f) o de $11 \mathrm{~mm}$ : face inférieure de la partie caudale du corps $\times 10$

g) $ᄋ$ de $9 \mathrm{~mm}$ (holotype): péréiopode II droite, face caudale $\times 35$

h) Carte de la région de la Kolpa et la Korana avec les stations de Monolistra caeca caeca et M. c. meridionalis.

12 et 3: grottes ou sources habitées par Monolistra c. caeca

45 et 6: grottes habitées par M. c. meridionalis

1. Sopot pećina, Severin na Kupi.

2. Source "Vrelo Okrugljak», Velika Jadić

3. Grotte de la "Vrelo Rudnica», Kamenica, Slunj.

4. Grotte: "Pećina jezero», Barilović, Karlovac

5. Grotte près de Tržić, (Mrežnica rijeka), Primišlje

6. Grotte "Crna peçina", Novi Kršlija, Rakovica. 


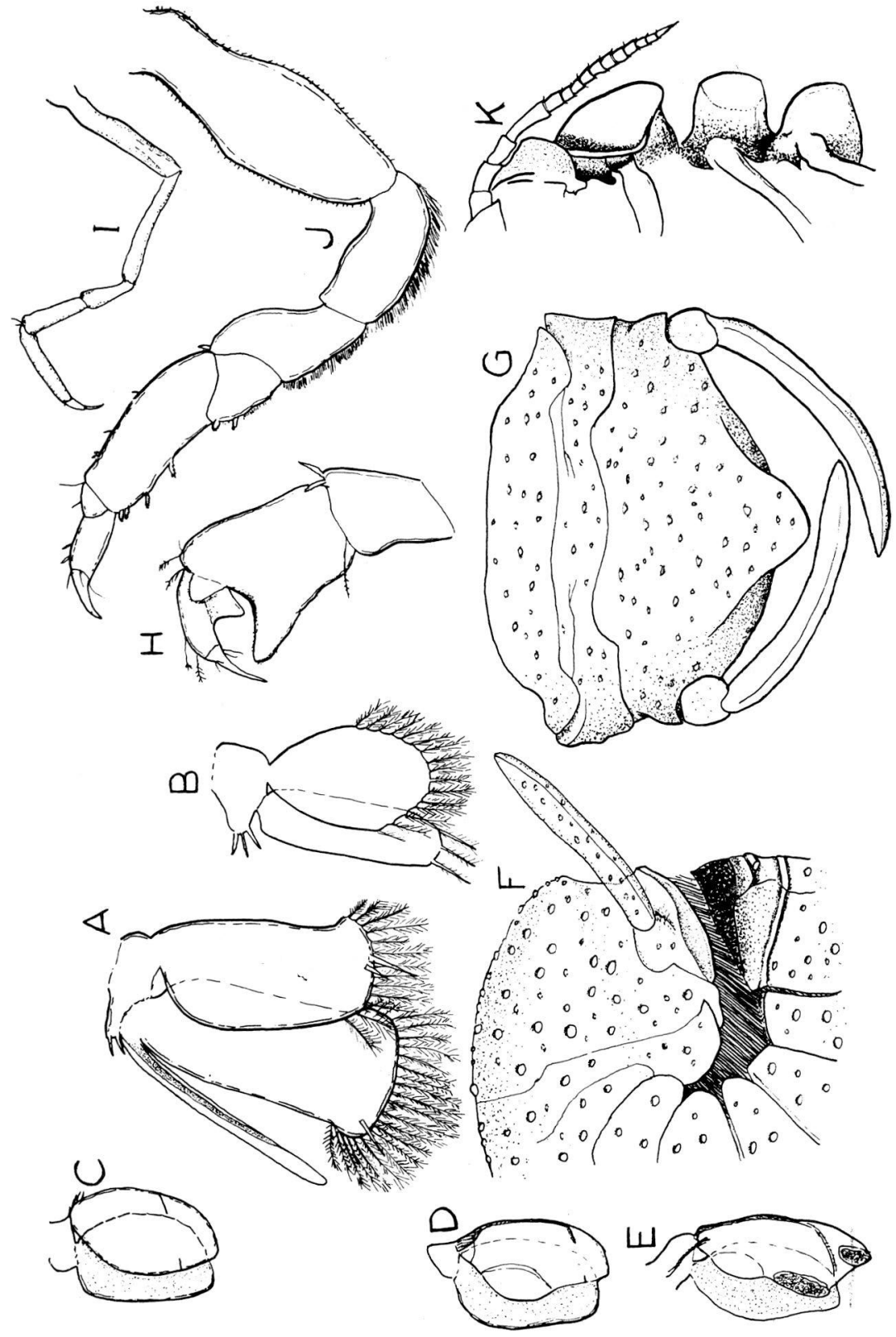




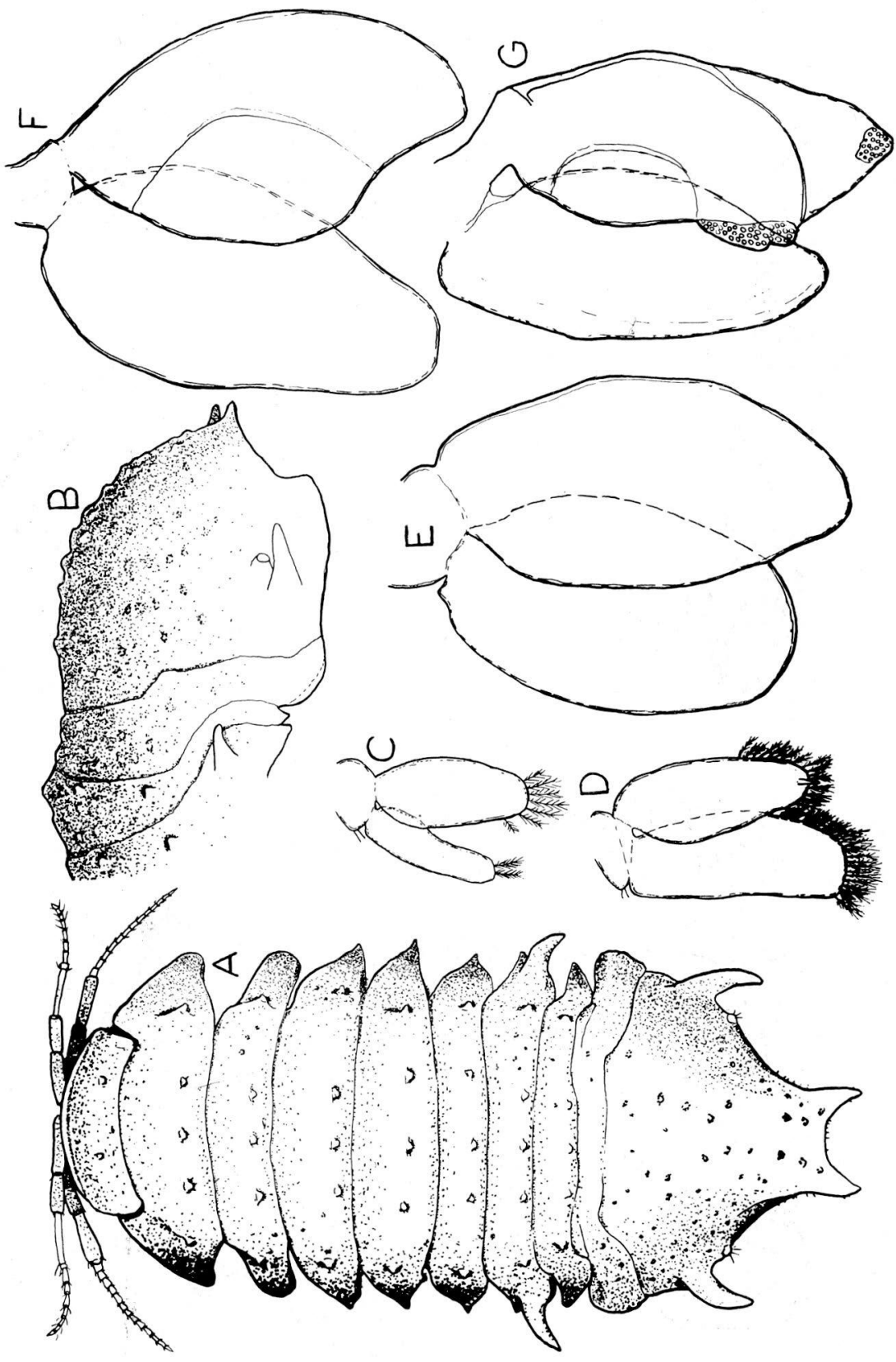


